interpretation are just as great. In the extreme case, when the results of trials are still being hotly debated (as, for example, in the question of patients with myocardial infarction), the findings of cost-effectiveness studies could become too controversial to be useful.

A distinction is usually drawn between cost-effectiveness and cost-benefit analysis. The former measures only the various effects of procedures, while the latter attempts to express the effects (benefits) in terms of money. It is at this stage that rarefied discussions of the value of life and health begin to dominate the reports. Though potentially useful frameworks have been developed, ${ }^{3}$ there is no agreement on how we should value the so-called "intangible" outputs of the Health Service. ${ }^{4}$ For this reason, cost-effectiveness analysis is the more practicable and useful procedure.

Ross et al have published data on the effectiveness of cardiac surgery on the quality of life. Some 20 months after operation $68 \%$ of patients reported no limitation on activity, and $74 \%$ of men were in full-time employment, compared with $12 \%$ and $33 \%$ respectively before operation. So, given that these comparisons genuinely reflect effectiveness, we may ask if cardiac surgery is good value for money. The answer will depend on comparison with alternative ways of spending it. Unfortunately, because of the problems referred to, we have few comparable data for other procedures. Perhaps renal transplantation is one other technique for which we have the most comprehensive follow-up data, for virtually the whole European experience is recorded in the computer files of the European Dialysis and Transplant Association. As a result, we can say with confidence that the output of renal specialists varies between about $£ 1000$ per year of "good quality" life (for patients with a functioning transplant) and $£ 11000$ per year of "poorer quality" life (for patients treated with hospital dialysis). ${ }^{6}$ Such confidence, however, is unusual.

Bunker et $a l^{7}$ have collected a series of cost-effectiveness studies of surgery from America and Britain. All these have made the best of such data that exist, with the aid of simplifying assumptions. Sometimes the results of the analysis are convincing. For example, Neutra, ${ }^{8}$ extrapolating data from White ${ }^{9}$ to the whole of America, estimated that careful observation and evaluation of all diagnostic information before appendicectomy could save 170 deaths, 6 million convalescent days, and $\$ 370 \mathrm{~m}$ in averted treatment costs per year. Appendicectomy lends itself to measurement of costs because the expected benefits (such as lives saved and reduced number of days with disability) are easily assessable immediately after operation. Furthermore, the results of this study were clear cut because the greater the care taken before operation the greater the benefit.

In many other cases the analysis can contribute little to any debate. Thus Bunker et al, ${ }^{7}$ considering the cost-effectiveness of elective hysterectomy, judged that the procedure had no significant effect on the expectation of life. So we have to alter the central question to the effect of the operation on the quality of life. But we then find no convincing data on benefits in these terms: on the one hand, the woman may lose her symptoms from fibroids or her menstrual discomfort; on the other hand, the operation itself may have unpleasant sequelae.

A constant theme running through all the discussions on cost-effectiveness studies is how fragmentary are the data on which calculations have to be based. Moreover, few of the controlled evaluative trials that have been done can be compared with one another. There is a strong case for closer co-operation between clinicians and economists, especially in large and expensive multicentre trials. Not only would this ensure that trials generate useful data: it would also mean that these produce results that are sufficiently standardised to allow us to make true comparisons between the alternative ways of spending money in the NHS.

${ }^{1}$ Cochrane, A L, Effectiveness and Efficiency. Random Reflections on Health Services. London, Nuffield Provincial Hospitals Trust, 1972.

${ }^{2}$ Monro, J L, et al, British Medical Fournal, 1978, 1, 1684.

3 Williams, A, British fournal of Preventive and Social Medicine, 1974, 28, 196.

4 Card, W I, and Mooney, G H, British Medical fournal, 1977, 2, 1627.

${ }^{5}$ Ross, J K, et al, Thorax, 1978, 33, 3.

${ }^{6}$ Office of Health Economics, Renal Failure, a Priority in Health? London, OHE, 1978.

${ }^{7}$ Bunker, J P, et al (ed), Costs, Risks and Benefits of Surgery. New York, Oxford University Press, 1977.

${ }^{8}$ Neutra, R, in Costs, Risks, and Benefits of Surgery, ed J P Bunker, B A Barnes, and F Mosteller. New York, Oxford University Press, 1977.

${ }^{9}$ Medical World News, 1975, 16, 21.

\section{Prognosis of cryptogenic fibrosing alveolitis}

The symptoms, signs, and functional effects of widespread pulmonary alveolar fibrosis are well recognised; and it is known to result from a large variety of antecedent processes, some of known but many of unknown cause. ${ }^{1}$ Among the latter is a sort of inflammatory change in pulmonary acini, differing from bacterial and viral pneumonias not only in the lack of an evident external cause but also in its histopathology and course. The inflammatory process predominantly affects the alveolar walls, and there is a strong and early tendency to fibrosis within them. This contrasts with the inflammation provoked by infections, typically leading to consolidation by exudation into alveolar spaces, which usually resolves-and, if it does not, undergoes fibrosis by organisation of intraalveolar exudate or, more rarely, suppurates. Differences between the two sides of the Atlantic have arisen over the terminology of these two types of inflammation in the lungs. ${ }^{3}$ On both sides, "pneumonia" without qualification would be taken to refer to consolidation. In North America inflammation predominantly affecting alveolar walls is called "interstitial pneumonia." In Britain the convention that "alveolitis" should be used for this kind of inflammation, first suggested in $1964,{ }^{2}$ has been generally adopted; "fibrosing" can be added to indicate the strong tendency to fibrosis, and "cryptogenic" if required to specify that it is of unknown cause.

Within the broad histological criteria for fibrosing alveolitis or interstitial pneumonia there are wide variations not only in such features as extent of fibrosis and distortion of structure, which are likely to be related to duration, but also in the relative prominence of and the types of cells concerned in alveolar wall thickening, hyperplasia of lining cells, and cellular content of alveolar spaces. Not surprisingly, patients with such diverse lung changes present varied clinical features, with differences in acuteness of onset, duration and course of illness, associated diseases, and response to corticosteroids. A dysfunction of the immune system as a factor in pathogenesis is suggested by several findings-the presence of rheumatoid and antinuclear factors in the serum of some patients ${ }^{45}$; the fact that their lymphocytes may produce lymphokines when exposed to collagen ${ }^{6}$; and the association in some cases with such diseases as rheumatoid arthritis, systemic sclerosis, Sjögren's syndrome, polymyositis, and hyperglobulinaemic renal tubular acidosis. There is no reason to suppose, how- 
ever, that lung changes of any of these histological patterns are uniquely related to any one causal factor or specifiable set of factors. Nevertheless, correlations between definable variants of the general pattern and clinical features, especially prognosis and responsiveness to corticosteroids, are obviously of practical importance.

In 1965 Liebow et $a l^{7}$ described a pattern which they called "desquamative interstitial pneumonia" (DIP), whose special features were large cells thought to be granular (type II) pneumocytes filling alveoli and lining them; relatively little thickening of alveolar walls; uniformity of the changes; prominence of lymphoid follicles; and absence of necrosis, hyaline membrane, and fibrin. Perhaps not surprisingly, patients who satisfied these criteria were found to have a better prognosis than those with "usual" interstitial pneumonia (UIP) not having these characteristics. In particular, the DIP patients included more with a good response to corticosteroid treatment. This correlation has been confirmed by others, ${ }^{89}$ But not all have accepted the implication that DIP is different in some way other than histological pattern from the "rag-bag" of UIP. 81011 And from electron-microscope studies most of the intra-alveolar cells in the DIP pattern are now generally agreed to be macrophages, ${ }^{10-14}$ with only a few type II pneumocytes. Thus the adjective "desquamative," suggesting that these cells are derived from alveolar lining cells, is misleading; but it has passed into general use, even by those who do not wish to commit themselves to the view that the histological pattern to which it refers delineates a pathogenetically distinct group.

Carrington et $a l^{15}$ have now reiterated the arguments for the separateness of DIP. They selected from 490 patients with diffuse lung disease seen between 1952 and 1976 who had had open lung biopsy (or, in a few cases, necropsy shortly after clinical study) 130 who had shown changes interpreted as some sort of interstitial pneumonia and had been followed for at least 12 months or until death. From these they further selected 40 that they classified confidently as DIP and 53 as UIP, adopting criteria for DIP similar to those of Liebow et $a l^{7}$ but omitting lymphoid hyperplasia. In the DIP group mortality during a mean period of $9 \cdot 1$ years was $28 \%$; improvement occurred after corticosteroid treatment in $62 \%$ and spontaneously in $22 \%$. In the UIP group, on the other hand, mortality during a mean period of 5.6 years was $66 \%$; only $12 \%$ improved after corticosteroid treatment, and none spontaneously. As compared with other reported series, the proportion classified as DIP is high; indeed, in this series DIP was quite a usual pattern. The decision between DIP and the uncategorised group must have been finely balanced in borderline cases; unfortunately, no information is given about the 37 patients not classified as either DIP or UIP.

While the association of a desquamative pattern with the possibility of response to corticosteroids or of remission has been generally accepted, we need to examine the implication that DIP should be regarded as a different disease from other forms of interstitial pneumonia and fibrosing alveolitis. In what other respect do cases placed in this category differ from those labelled "usual"? Correlations between variations in histological pattern and clinical course do not necessarily imply differences in causation, since they can be observed within aetiologically specific diseases such as tuberculosis and syphilis. But it is doubtful whether Carrington et $a l^{15}$ have aetiological differences in mind, since they comment that "UIP was considered to be an etiologically non-specific reaction, and it was suspected that DIP might also have more than one cause." Being aetiologically non-specific and having more than one cause would usually be regarded as synonymous; but perhaps these different phrases are intended to $\underline{T}$ imply that the range of causal factors in DIP is restricted, and 3 possibly exclusive of a wider range concerned with UIP. Even $\stackrel{\mathbb{Q}}{2}$ this moderate statement is opposed by the observation that $\stackrel{\complement}{\leftarrow}$ there was no correlation between histological pattern and $\vec{F}$ association with possibly related diseases: "collagen vascularo diseases" accompanied $13 \%$ and $21 \%$ of cases classified as $\underline{\bar{O}}$ DIP and UIP, and two classified as UIP and one as DIP were $\frac{\overline{\bar{T}}}{\bar{\sigma}}$ thought to be reactions to drugs.

Those who wish to avoid using terms referring to histological patterns in such a way that they appear to imply $\vec{\circ}$ pathogenetic differences are said by Carrington et $a l^{15}$ to regard DIP and UIP as "merely early and late patterns of a $\vec{\omega}$ single disorder." Such a view would be as unjustifiably dogmatic as is that suggested by Carrington et al, which implies relationships between histological patterns and assumed but unidentified pathogenetic differences; and it may be $\vec{A}$ doubted whether anyone holds it. Certainly, discussions of such correlations are likely to be profitable only after causal $\stackrel{\infty}{+\infty}$ factors have been identified. ${ }^{18}$

The information derived from lung biopsy may be required for diagnosing difficult cases of fibrosing alveolitis and helps $\omega$ in recognising patients likely to respond to corticosteroids. $\mathbb{D}$ But some British workers have believed that in many cases, $\frac{\mathbb{D}}{3}$ especially of the more chronic type, diagnosis can be reached픙 with sufficient assurance for practical management without $\frac{\mathbb{D}}{-}$ open lung biopsy, and that management can be based on $\vec{\theta}$ observation of the effect of corticosteroids. On this view 0 assessing the initial response is part of the investigation, and even if lung biopsy shows a predominantly "mural" (or "usual") pattern they argue that the patient's interests would be best served by a carefully assessed trial of such treatment. This view was supported by evidence that the early response $\varrho$ to corticosteroids is correlated with survival. ${ }^{9}$ This has $\overrightarrow{\overrightarrow{0}}$ recently been confirmed ${ }^{16}$ in a study of 127 patients in whom response was assessed six weeks after treatment was started: mortality within two years of presentation was $23 \%$ in those with a good response and $56 \%$ in those with a poor or no response.

Lung biopsy, correlated with intensive clinical, radiological, $\frac{0}{3}$ physiological, and immunological study, has added greatly to knowledge of this difficult condition. But we must distinguish between investigations desirable for increasing knowledge and those required for the management of individual patients. One benefit of increased knowledge may be a diminished need $\stackrel{N}{T}$ for invasive diagnostic procedures.

${ }^{1}$ Scadding, J G, Thorax, 1974, 29, 271.

2 Scadding, J G, British Medical fournal, 1964, 2, 686.

3 Scadding, J G, British fournal of Diseases of the Chest, 1978, 72, 1.

Turner-Warwick, M, and Doniach, D, British Medical fournal, 1965, 1, 886.

5 Turner-Warwick, M, and Haslam, P, Clinical Allergy, 1971, 1, 83.

${ }^{6}$ Kravis, T C, et al, fournal of Clinical Investigation, 1976, 58, 1223.

Liebow, A A, Steer, A, and Billingsley, J G, American fournal of Medicine, 1965, 39, 369.

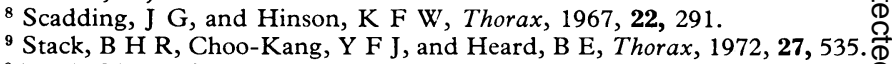

10 Patchefsky, A S, et al, Thorax, 1973, 28, 680.

11 Valdivia, E, et al, Thorax, 1977, 32, 7.

${ }^{12}$ Brewer, B D, Heath, D, and Asquith, P, fournal of Pathology, 1969, 97, 317.

${ }_{13}$ Shortland, J R, Darke, C S, and Crane, W A J, Thorax, 1969, 24, 192

${ }^{14}$ Farr, G H, Harley, R A, and Hennigar, G R, American fournal of Pathology $1970,60,347$

15 Carrington, C B, et al, New England fournal of Medicine, 1978, 298, 801."

${ }^{16}$ Johnson, A J, et al, Thorax, 1977, 32, 650. 